\title{
Second Screen Comes to the Silver Screen: A Technology Feasibility Study Regarding Mobile Technologies in the Cinema
}

\author{
Stuart Cunningham and Jonathan Weinel \\ Creative and Applied Research for the Digital Society (CARDS) \\ Glyndŵr University \\ Wrexham, North Wales, UK \\ \{s.cunningham $\mid$ j.weinel\}@glyndwr.ac.uk
}

\begin{abstract}
The act of 'second screening' involves the use of an additional media screen such as provided by a mobile phone or tablet, to consume content alongside a primary screen such as a TV. In this paper we present findings of a study that explored the feasibility of using second screen technology in a cinema environment. A proposal for the application of second screening within the cinema context is discussed. Following this, the feasibility of the proposed design is considered in relation to available technologies, a consumer study and a market survey.
\end{abstract}

Keywords-Context-aware computing, Affective computing, Second screen, Internet of Things, Cinema

\section{INTRODUCTION}

This study investigates the feasibility of utilising 'second screen' technology within the context of the cinema. Specifically, we were interested in whether a mobile device could be used to provide synchronised features that would enhance the experience of viewing a movie at the cinema. Among the motivating factors to explore this area was the fact that many consumers have been losing interest in actually attending the cinema over recent years; this is due to a variety of factors that include the rise in streaming media services, illegal downloading and expensive ticket prices. At the same time, the consumption of content via mobile phones has become a pervasive feature of modern society. Therefore, applying second screen technology within the cinema was seen as a possible means to enhance the user experience and provide a richer experience than is possible at home, thereby helping to reinvigorate the industry.

In order to explore this area, a design for a second screening technology for use within the cinema was proposed, and evaluated through a review of technology, a consumer survey, a market survey and two case studies. The outcomes of the study enable us to comment on the extent to which second screening within the cinema context may appeal to consumers, and which aspects of such a technology are likely to be received positively or negatively.

\section{BACKGROUND}

In the UK, social networking and mobile technologies continue to grow in popularity. $39 \%$ of adults own smartphones [14]. 73\% of 16-24 year olds are using social networking, and about half of them use mobile devices to communicate with their friends about TV programmes they are watching [8]. This is 'second screening': the use of a mobile device such as a smartphone or tablet computer to carry out other activities while another screen shows the 'main' content - a TV programme for instance.

Second screening is a 'hot topic' in media and advertising, as it presents a new way to capture audience attention. The media industry is likely to grow to a value of $£ 63$ billion by 2016 [16]. Part of this growth will be in Wales where, recent investment from Virgin Media has created new jobs in Swansea [4]. People are increasingly exercising greater choice over how and where they consume content through mobile devices. This is seen not just as an advertising opportunity, but also in some cases a necessity: TV companies do not want to lose their customers to the second screen. Harnessing second screen allows businesses to target advertisements and products to the consumer, but there is also the potential to synchronise the content so that it compliments the primary screen and encourages its use.

These are the principles behind the commercial drive for second screening, but the reality when looking at the available second screen apps falls somewhat short of this. The range of second screen apps available has been described as 'the wild west' [24]; many different companies are attempting to 'cash in', but the result for the consumer is rather cluttered, with few unified solutions, as we shall see. A few apps like Zeebox [26] are beginning to take the stage, offering second screening services to cover many different TV programmes. Nonetheless, most of the young adults we spoke to during this study were not users of purpose-built second screen 
apps; instead they used their phones for texting, email, general web-browsing and Facebook. Only one person from 89 respondents said they used Zeebox. Most had not actually heard the term 'second screening', though they admitted to engaging in this type of activity.

Second screening for films on TV or through home media has some precedent as we shall see, but has yet to hit the movie theatres during performances. We already know that young people are second screening in front of the TV, but we don't know if there is demand for this to happen in the cinema from consumers or from the industry. This feasibility study seeks to investigate this, with a view to potentially developing the proposed technology for use in some of the 745 UK cinemas [5].

\section{Proposed Technology}

Figure 1 shows the proposed design for a second screening technology within a cinema context. The model indicates the use of a second screen to exchange information via various sources. These may include: audience polls, where information is exchanged with the venue, and to other audience members; additional film resources, these could be similar to 'DVD extras', or other content that compliments the film; merchandising and information regarding the cast and crew, via the Internet. The proposed design therefore suggests the synchronous use of a mobile phone to access additional content during the film, and implies network communication between the film and the device in order to tailor available content to the events on-screen as they happen.

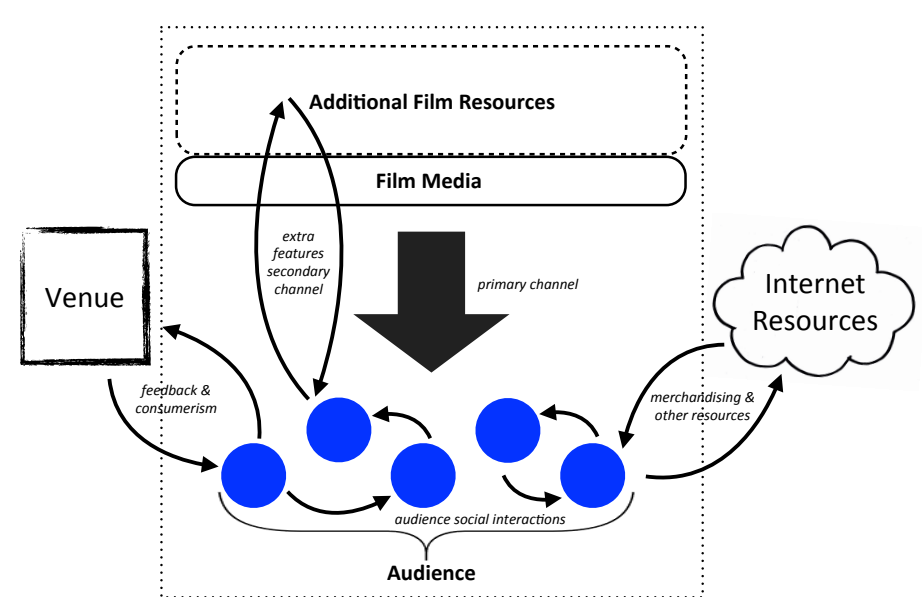

Figure 1. Theoretical model in cinema context.

\section{TeChNOLOGY REVIEW}

\section{A. Existing Software}

In this section we consider an overview of existing technologies which use second screening, or which offer forms of human-computer interaction, which are broadly similar to second screening.

\section{1) Cinema Apps}

A variety of asynchronous cinema apps exist which enable users to search for film times, book tickets, view trailers and locate local cinemas. These include apps from cinema chains, including: Odeon, Cineworld, and Vue.

While the above apps are all asynchronous, in the US National Cinemedia's app Movie Night Out with CinemaSync [13] offers targeted services such as games for the user during the pre and post movie experience in the cinema lobby. Screenvision's Screenfanz [17] app similarly targets the pre-show situation, with advertising, movie listings, social features (such as user reviews), games and in-theatre quizzes. The UK has followed with the CiniMe [7] app for pre-show entertainment.

\section{2) Film Apps}

Considering film-related apps, audiences can use IMDb Movie \& TV [11] to search a database for cast information, user reviews, plot synopsises, trailers, etc. In addition, various film-specific promotional apps are available such as The Amazing Spider-Man AR [19], which uses the phone camera to scan movie-themed images and unlock corresponding animations. Happy Feet Two: The Penguin App [21] similarly provides a penguin animation that responds to touch actions and voice.

While most promotional film apps are asynchronous, a few such as the Prometheus - Weyland Corp Archive Second Screen App [10] provide additional content similar to DVD/Blu-ray 'extras', and also offer a synchronised mode that can be used to provide the relevant content such as deleted scenes in parallel with the movie, when viewed at home. Marvel's the Avengers: A Second Screen Experience (Marvel Entertainment, 2012) and The Dark Knight Rises FX HD [22] offer a similar synchronised experience for home audiences. Disney's Second Screen apps for iPad [9] are another significant example. The various apps offer synchronised second screening facilities for a range of Disney films.

\section{3) TV Second Screen Apps}


TV presents a much larger market for advertising than film; as the BFI indicates [5], in terms of UK film viewing alone $77 \%$ takes place through the $\mathrm{TV}$, while only $3.4 \%$ happens in the cinema. Various existing apps have begun to emerge which attempt to harness the advertising capabilities of second screen for TV, some of which offer synchronous features of the type under discussion. Zeebox for example, provides additional information, content and services related to TV [26]. BSkyB, NBCU and Comcast are stakeholders in the company, indicating the significant corporate interest in second screening for TV [3]. Notably, the app is also internationally supported (UK, US, Australia) and spans multiple operating systems (iOS, Android). In the UK, viewers can select from multiple digital TV services as provided by Freeview, Sky and Virgin (for example). Another second screen app for TV: Viggle provides a reward system for viewers, who can earn points by watching programmes and redeem these against various items such as movie tickets, music, gift cards or charity donations [20]. Similarly, GetGlue provides social features and a reward system for TV audiences [1]. The latest versions of Shazam [18] (an app which uses audio fingerprinting to provide additional content related to music), can also identify TV programmes and provide relevant content. Yahoo's IntoNow [25] uses a similar audio fingerprinting technology, providing a variety of features such as posting to social media sites and direct links to buy the music heard in the programme.

The examples discussed are some of the most significant second screening apps for TV, which are applicable for multiple programmes, however there are also many other programme-specific apps for shows such as Lost, Breaking Bad, Dexter or the Big Band Theory which are similar to those discussed for film and provide asynchronous content. There are few synchronous examples, although Channel 4's Facejacker [23] uses Intrasonics' inaudible audio tagging technology to synchronise the app with the TV programme and push the relevant content [6].

Competition for dominance on the second screen market has been described as strong, but with currently a disparate range of apps and solutions on the market [24]. Nonetheless, recent developments may indicate a convergence towards multi-programme, cross-platform, synchronous apps.

\section{4) Ad-hoc Second Screen Apps}

Second screening can also take place in a more general/ad-hoc fashion through a wider variety of other popular apps that are available: Facebook and Twitter being key examples. These apps are not explicitly related to particular TV programmes or films, however the social facilities they provide enable audiences to discuss any programming, before, during or after it occurs. The significance of these social media second screening solutions should not be overlooked. As a Deloitte report regarding the TV market indicates: while about half of 16-24 year olds occasionally or frequently discuss what they are watching via messaging, email, Facebook or Twitter, two thirds of those with second screen devices stated that they did not want to use them to interact with programmes [8].

The findings above have some implications that are relevant to consider in relation to the proposed technology. Firstly, social interactions form a significant aspect of second screening, and are probably worthwhile to include in any design. Our web survey (discussed later), supports this, but found that information and extra features related to the movie were more popular feature suggestions. Secondly, in the TV setting the second screen is not necessarily used for purposes directly related to a 'first screen'. If these behaviours were reflected in the cinema setting, there would be only limited interest in a second screen app that provided related content, and an app that mediated activity could be seen as a restrictive by audiences.

\section{5) Smart TV Apps}

Smart (or 'connected') TVs and EPGs (electronic programming guides) provide another means through which consumers may use apps and additional content. While these are not 'second screens' in the sense that the content is usually viewed on the same screen, they can provide similar functions. The most popular apps for connected TVs are currently those that provide streaming services such as Netflix or LoveFilm. Netflix has an associated app for smartphones, which can be used either for viewing the films, or to remotely control what the service displays on other devices (e.g. smartTV or games console). Though the take-up of connected $\mathrm{TV}$ has been slow, many people have access to a device that can be used to connect a TV [8]; games consoles and laptops are examples.

\section{B. Existing Facilitative Technology}

This section looks at the available technologies that could be used for the proposed project. App development can be carried out using typical tools and methods; it is not necessary for this report to reiterate these, except to highlight that developing for multiple devices and operating systems is likely to increase the 
number of potential users (and hence, profitability) of the technology. There are however some further specific technology considerations for the proposed project, such as those arising from the need for synchronisation between the app and the external media (in this case, a film). This section will explore possible technologies that can be used to achieve this synchronisation. Additional aspects, such as approaches that enable configuration for small businesses, may also be considered.

\section{1) Location Services}

Location services are a common feature of many modern smartphone handsets and tablet computers. As discussed previously, other apps enable the use of these locationbased services to target the content provided within the app to the user when they are in the cinema. The existing technologies for this are already in place and widely available, so this provides one possible method that can be utilised by the proposed second screen technology. Location services could be used to identify when the user arrives at a movie theatre, for example.

The accuracy of location services varies depending on a variety of factors; for example: known locations of cell towers, WiFi and GPS. It is unlikely that location services will provide sufficient accuracy to identify the specific activities of the user once they are indoors in a cinema (for example, to identify if they are in a lobby or a particular screen), since line of sight to GPS satellites would be lost. This limits the potential of GPS to enable synchronisation with a movie, when used in isolation. However location services could be used alongside one or more other methods to enable this. For example, GPS could be used to identify when the user gets to a cinema. The app could then prompt the user to specify which performance they will be watching. Such approaches would be viable since cinemas provided scheduled performances, and the number of concurrent performances is limited by factors such as the number of screens.

\section{2) Audio Watermarking}

As discussed previously, audio watermarking is used by Intrasonics to provide synchronisation between apps and TV programming. This works by providing inaudible (to the human ear) trace codes within the programme's audio stream, which the app can detect and decode to push the desired content in synchronisation. Intrasonics own the patents for this technology, which is commercially licenced to developers. Therefore this may not be a suitable method of obtaining synchronisation for the proposed project, due to the licencing costs involved.

In addition, audio watermarking requires the relevant codes to be incorporated in the media that is to be synchronised. This would require the co-operation of film producers, which may be difficult to obtain for a majority of mainstream movies. It may be possible to arrange such co-operative development for isolated cases and performances, similar to the App movie example.

Intrasonics technology might be a suitable solution for the proposed technology, but the licencing costs and the required co-operation of movie production studios may be prohibitive factors for this project.

\section{3) Audio Fingerprinting}

This is distinct from audio watermarking, since it relies on reference libraries. In this scenario the app 'listens' for a recognisable audio fingerprint, which it compares to a library to identify the media. This technology might be a possible solution to obtain synchronisation via audio, and has the benefit that the audio signals do not need to be embedded in the films beforehand. This method would allow accurate synchronisation with films, however the audio fingerprinting approach is covered by patents owned by Shazam and SoundHound, which is likely to be prohibitive for the proposed project.

\section{4) Radio Frequency Identification (RFID)}

RFID enables identification of objects or entities (the system can also be used on animals and people, for example) using radio-waves. The approach requires a RFID chip to be attached to, or embedded in the object to be tracked. An RFID reader is required to scan the data. The technology is currently employed in a range of applications in areas such as asset tracking, access control and payment systems [15]. Examples of use include Tesco, who use it for supply chain management, or the Oyster card system in London, which uses it for payment. In mobile devices, RFID is utilised in a standardised form through Near Field Communication (NFC), which enables close-range scanning.

For the proposed project, NFC could be used to synchronise mobile devices with movie performances. Chips could be located at the entrance to movie screens, or in the seats of the theatre; the specifics of implementation would be a subject for further investigation during the design phase of the project. Many mobile devices that are already on the market incorporate NFC readers. It has been suggested that the 
majority of mobile devices could have NFC readers built-in within 5-10 years [15]. Notably, Apple's iPhone does not yet incorporate NFC, and can only be used as an RFID reader through the addition of accessories. A patent issued to Apple for a technology similar to NFC suggests that the company may attempt to develop their own alternative technology.

Since NFC is already available in many mobile devices, with indication that this will increase, it may be a suitable choice for the proposed second screen technology. The technology would provide suitable synchronisation possibilities for film applications. It is also likely to be an effective solution if the proposed project was developed for other applications, such as zoos, historical sites etc., since RFID tags could be installed in other types of objects (or indeed, animals). While this study focuses on the cinema application, it is worth mentioning that other methods explored in this section may be less suitable for other situations. For example, a historical site is unlikely to be able to use audio watermarking or fingerprints, but could easily make use of RFID tags.

\section{5) Integrating Social Features}

Many existing apps integrate aspects of Twitter or Facebook. It should not present any particular difficulties for the proposed technology to integrate with these social apps. The proposed technology could for example, push information to Facebook or Twitter.

\section{6) Syncing Time Codes}

One possible approach to synchronisation could be to sync the timecodes associated with video projection equipment, with mobile devices over wifi networks. Cinemas use a computer-based play-listing system ${ }^{1}$ that is connected to a network, to play films, adverts and precisely time-synchronise other events such as the dimming of lights. This system could therefore be used to provide precise synchronisation of mobile devices with events in the cinema, through use of a Wi-Fi connection. As the cinema is a fixed environment that accommodates specialist equipment, required software or hardware installation could be made as necessary. This potential should make it easier to synchronise content effectively without the need for the audio fingerprinting or watermarking techniques used in home environments where the equipment used may be variable.

1 The function of this system was reviewed during our visit to the Scala cinema as part of the case study component of this investigation.

\section{7) Face Detection}

Face Detection technology is readily available in cameras, and can be used to identify faces which a camera is pointing it. It should be possible to adapt this technology to identify actors on screen, particularly if used in combination with synchronised timecodes (since the film timecode could be compared with an on-screen image).

\section{8) Configurations}

Previous discussion indicates that support for as many venues and situations as possible will increase the value of the proposed technology. This could be considered in terms of 'the long tail' model [2], which describes the business value (as used by companies such as Amazon) in catering for a large number of niche requirements. The model describes the combined benefit of catering for such niche requirements, which individually might be seen as insignificant. For Amazon, the largest share of their profit is produced by providing access to a vast range of specialist interest books. Seen in isolation, and in comparison with 'best sellers' there might be little perceived benefit to selling any one of these specialist titles, yet the sum total of all books sold as niche interest sales outweighs the best sellers.

In the context of cinemas, in Wales alone there are over 50 small independent and community-run venues. When looking more broadly at the possible application of the proposed technology for small businesses and industries in other sectors (zoos, museums, etc. ${ }^{2}$ ), we can apply the long-tail model. Thus the technology becomes much more useful if it can be applied for the smallest, least technically savvy or financially supported business (as opposed to only catering for major chains or large scale operations).

In order to facilitate this, the proposed technology should be easily customisable for multiple users/venues, a web based system could be used that enables the user to upload personalised content and update basic features, without having to manage graphical or functional issues. This could be accomplished using the approaches that have become standard in web 2.0 tools for blogging etc. Therefore, it should be possible for almost any individual with basic ability (e.g. able to use Facebook to create an event page) to make use of the system.

\section{9) Google Glass}

\footnotetext{
2 The comments provided here relate primarily to revised design 2 of the technology outlined at the end of this document.
} 
The forthcoming Google Glass technology will enable the user to use a head-mounted display. This technology could be adapted for the proposed project, when it becomes available. The particular advantage for the proposed project is the potential for a more discrete, less intrusive second screen, since many of those interviewed as part of this study expressed concern over issues such as the distraction caused by light from mobile screens. This could be reduced using technology such as Google Glass, since only the individual (wearer) would be able to see the screen.

\section{CONCLUSION}

In conclusion, our study shows that second screening within the cinema is technologically possible, and that synchronous content could be provided using WiFi, RFID chips or audio watermarking.

Future work will consider the feasibility of the concept in the marketplace and likely uptake and attractiveness to commercial cinemas.

\section{REFERENCES}

[1] AdaptiveBlue (2013). GetGlue (Version 4.0) [Mobile application]. Available https://itunes.apple.com/gb/app/getglue/id377615302?mt=8 (Accessed 18 February 2013).

[2] Anderson, C. (2004). 'The Long Tail'. Wired 12(10). Available at: http://www.wired.com/wired/archive/12.10/tail.html (Accessed 27 March 2013).

[3] Andrews, R. (2013). 'NBCU, Comcast buy in to Zeebox to take 'second screen' to prime time'. paidContent. Available at: http://paidcontent.org/2012/09/27/nbcu- comcast-buy-in-to-zeebox-totake-second-screen-to-prime-time/ (Accessed 18 February 2013).

[4] BBC (2013). BBC News - Virgin Media to create 230 jobs in Swansea. Available online at: http://www.bbc.co.uk/news/uk-wales-21465618 (Access 21 February 2013).

[5] British Film Institute (2012). BFI Statistical Yearbook 2012. Available at: http://www.bfi.org.uk/statisticalyearbook2012/ (Accessed 18 February 2013).

[6] Channel 4 (2012). 'Channel 4 scores Facejacker app first'. Channel 4. Available at: http://www.channel4.com/info/press/news/channel-4scores-facejacker-app-first (Accessed 18 February 2013).

[7] CiniMe (2015). Cinime. Available at: http://www.cini.me/ (accessed 13 March 2015).

[8] Deloitte (2012). TV: Why? Perspectives on TV: dual screen, catch-up, connected TV, advertising, and why people watch TV. Available online at: $\quad$ http://www.deloitte.com/view/en_GB/uk/industries/tmt/mediaindustry/perspectives- on-the-uk-tv-sector/index.htm (Accessed 18 February 2013).

[9] Disney (2013). Disney Second Screen [Online]. Available at: http://disneysecondscreen.go.com/ (Accessed 18 February 2013).

[10] Fox Digital Entertainment, Inc. (2013). Prometheus - Weyland Corp Archive Second Screen App (Version 1.0.5) [Mobile application].
Available at: https://itunes.apple.com/gb/app/prometheus-weyland-corparchive/id563562816? $\mathrm{mt}=8$ (Accessed 18 February 2013).

[11] IMDB (2013). IMDb Movies \& TV (Version 3.0.2) [Mobile application]. Available at: https://itunes.apple.com/app/imdb-moviestv/id342792525? mt=8 (Accessed 18 February 2013).

[12] Marvel Entertainment (2012). Marvel's the Avengers: A Second Screen Experience (Version 2.1) [Mobile application]. Available at: https://itunes.apple.com/gb/app/marvels-avengers-secondscreen/id539555261?mt=8 (Accessed 18 February 2013).

[13] National Cinemedia, LLC (2013). Movie Night Out with Cinema Sync (Version 3.2.1) [Mobile application]. Available at: https://itunes.apple.com/us/app/movie-night-out-

cinemasync/id382738878? $\mathrm{mt}=8$ (Accessed 18 February 2013).

[14] Ofcom (2012). Ofcom Communications Market Report 2012. Available online http://stakeholders.ofcom.org.uk/binaries/research/cmr/cmr12/CMR_UK _2012.pdf (Access 21 February 2013).

[15] Pathak, R., Joshi, S., Parandkar, P., Katiyal, S., Ludhiyani, A. (2010). 'Applications of RFID and a software framework for facilitating its integration in mobile phones'. International Journal of Academic Research. 2 (5).

[16] PwC (2012). Entertainment \& media outlook: The outlook for the Entertainment and Media industry to 2016. Available online at: http://www.pwc.co.uk/entertainment- media/issues/entertainmentmedia-outlook.jhtml (Access 21 February 2013).

[17] Screenvision (2012). Screenfanz (Version 1.3.2) [Mobile application]. Available https://play.google.com/store/apps/details?id=com.screenvision.screenfa nz\&hl=en (Accessed 18 February 2013).

[18] Shazam Entertainment Limited (2013). Shazam [Mobile application]. Available https://play.google.com/store/apps/details?id=com.shazam.android\&hl= en (Accessed 18 February 2013).

[19] Sony Pictures (2012). The Amazing Spider-Man AR (Version 2.2) [Mobile application]. Available at: https:/itunes.apple.com/gb/app/theamazing-spider-man- ar/id518757797?mt=8 (Accessed 18 February 2013).

[20] Viggle (2013). Viggle (Version 1.4.1) [Mobile application]. Available at: https://itunes.apple.com/us/app/viggle/id487066871?mt=8 (Accessed 18 February 2013).

[21] Warner Bros (2012). Happy Feet Two: The Penguin App [Mobile application]. Available at: https:/itunes.apple.com/gb/app/happy-feettwo-the-penguin- app/id476702540? $\mathrm{mt}=8$ (Accessed 18 February 2013).

[22] Warner, Bros (2012). The Dark Knight Rises FX HD (Version 2.3) [Mobile application]. Available at: https://itunes.apple.com/gb/app/thedark-knight-rises-fx- hd/id541313964?mt=8 (Accessed 18 February 2013).

[23] Widebeam Digital Ltd. (2012). Facejacker (Version 2.1) [Mobile application]

Available https://itunes.apple.com/gb/app/facejacker/id498514693?mt=8 (Accessed 18 February 2013).

[24] Winslow, G. (2012). 'Broadcasters Sync Up Second Screen Efforts'. Broadcasting \& Cable, 19 November 2012. p20-21. Also available online at: http://www.broadcastingcable.com/article/490467Broadcasters_Sync_Up_Second_Screen_Efforts.php (Access 18 February 2013).

[25] Yahoo Inc (2013). IntoNow - Connect with your friends around the shows you love. Available at: http://www.intonow.com/ci (Accessed 18 February 2013).

[26] Zeebox (2013). Zeebox (Version 2.9.0) [Mobile application]. Available at: https://itunes.apple.com/gb/app/zeebox/id454689266? $\mathrm{mt}=8$ (Accessed 18 February 2013). 